Arch Dermatol Res (1986) 278:449-453

\title{
Increased aggregation and arachidonic acid transformation by psoriatic platelets: evidence that platelet-derived 12-hydroxy-eicosatetraenoic acid increases keratinocyte DNA synthesis in vitro*
}

\author{
K. Kragballe and J. D. Fallon \\ Department of Dermatology, University of Michigan Medical School, Ann Arbor, Michigan, USA
}

Summary. Certain archidonic metabolites may play a pathogenic role in psoriasis. Platelets are rich sources of 12-hydroxy-eicosatetraenoic acid (12-HETE) and thromboxane $A_{2}$, mediators of skin inflammation and platelet aggregation, respectively. We have studied untreated psoriatic patients without a history of diabetes mellitus and smoking. In psoriatics, platelet aggregation elicited by thrombin, ADP, and ristocetin was significantly enhanced as compared with healthy adult volunteers. Enhancement of platelet aggregation was detected in patients with both minimal and widespread disease. The formation of 12-hydroxy-5,8,10-heptadecatrienoic acid (HHT), a cyclooxygenase product, and 12-HETE, a 12-lipoxygenase product, was increased in psoriatics with widespread disease but not in those with minimal disease. Formation of 12-HETE was stimulated to a higher degree $(125 \%)$ than HHT $(98 \%)$ in psoriasis $(P<0.05)$. Addition of platelet-derived 12-HETE to cultured human epidermal keratinocytes resulted in a stimulation of the DNA synthesis $\left(68 \%\right.$ at $\left.10^{-7} M\right)$. These data suggest that platelet activation occurs in psoriasis, and that release of inflammatory and mitogenic compounds by activated platelets may play a role in the pathophysiology of psoriasis. Whether enhanced platelet aggregation in psoriasis is associated with occlusive vascular disease needs further investigation.

Key words: Arachidonate metabolites - Psoriasis Platelet aggregation - DNA synthesis

\footnotetext{
* An abstract of this work was presented at the meeting of The Society for Investigative Dermatology, Washington D.C., May 1984

Offprint requests to: Dr. Knud Kragballe, Dermatologisk afdeling, Marselisborg Hospital, DK-8000 Aarhus C, Denmark
}

\section{Introduction}

Increased lipoxygenation of arachidonic acid has been implicated in the pathophysiology of psoriasis [12]. Among lipoxygenase products, 12-hydroxy-5,8,10,14eicosatetraenoic acid (12-HETE) is present in the highest amounts in psoriatic lesions [10]. 12-HETE is chemotactic to neutrophils in vitro [7] and topical application of 12-HETE to normal human skin results in an erythematous response characterized by a neutrophil and mononuclear cell infiltrate [6]. Therefore, 12-HETE may be one of the mediators of the leukocyte infiltrate characterizing psoriasis.

While 12-HETE may contribute to the pathogenesis of psoriasis, the cellular source of 12-HETE in the psoriatic lesion has not been defined. Normal human epidermis produces predominantly 12-HETE among lipoxygenase products $[11,15]$. On the other hand, the neutrophil also present in psoriatic epidermis displays abundant 5-lipoxygenase activity, but no or little 12lipoxygenase activity [2]. In contrast, platelets can produce substantial amounts of 12-HETE [9]. Activated platelets may, therefore, contribute to the abnormal levels of 12-HETE in psoriatic skin. An activation of platelets might also make psoriatics more prone to occlusive, vascular disease, the incidence of which has been reported to be high in psoriatics [19].

In this study, we present evidence that aggregation and arachidonic acid metabolism of platelets are stimulated in psoriasis and that platelet-derived 12HETE increases the DNA synthesis of cultured human keratinocytes.

\section{Materials and methods}

Patients

Peripheral venous blood was drawn from 15 adult psoriatics (10 males and 5 females) who had not been treated for at least 2 weeks. Patients were divided into two groups based on whether they had a skin involvement of more or less than $10 \%$ of the body 
surface. Patients taking nonsteroidal antïnflammatory drugs, smokers, and diabetics were all excluded. Fifteen healthy adult volunteers (11 males and 4 females) with an age range similar to that of the psoriatics served as volunteers.

\section{Platelets}

Sodium EDTA blood was centrifuged at $120 \times g$ for $15 \mathrm{~min}$ to obtain platelet-rich plasma, which was further centrifuged at $1000 \times g$ for 15 min to pellet the platelets. Platelets were resuspended in PBS without $\mathrm{Ca}^{2+}$ or $\mathrm{Mg}^{2+}(\mathrm{pH} 7)$, counted in a hemocytometers, and diluted to $2 \times 10^{8} \mathrm{cells} / \mathrm{ml}$.

\section{Arachidonic acid metabolites}

Suspended platelets were mixed with $2 \mu M$ arachidonic acid (Sigma) and $2 \mathrm{mM} \mathrm{Ca}{ }^{2+}$ for $5 \mathrm{~min}$ at $37^{\circ} \mathrm{C}$ and then incubated with $1 \mu M$ A23187 (Calbiochem) or $10 \mathrm{U} / \mathrm{ml}$ thrombin for $15 \mathrm{~min}$ at $27^{\circ} \mathrm{C}$. Arachidonic acid and A23187 were diluted in ethanol (final concentration $<0.2 \%$ ). The incubation was stopped by adding 2 vols ice-cold methanol. After removal of denatured proteins by centrifugation at $600 \times g$ for $15 \mathrm{~min}$, lipids were extracted using a modification of the method described by Luderer et al. [17]. Samples were acidified to pH 3 with $2 \mathrm{~N}$ formic acid, applied to octadecylsilyl (ODS) silica columns (Waters Assoc.), and eluted sequentially with water, petroleum ether, and methanol under vacuum. The methanol fraction was analyzed using reversed-phase high-performance liquid chromatography (RP-HPLC) (Hewlett Packard 1090 liquid chromatograph) on a Hypersil ODS column $(100 \times 2.1 \mathrm{~mm})$ eluted with acetonitrile $(\mathrm{ACN})$ in aqueous phosphoric acid $(\mathrm{pH} 3)$. The mobile phase was $20 \%$ ACN with a linear increase to $30 \%$ over $2 \mathrm{~min}$ followed by linear increases to $33 \%$ from 2 to $10 \mathrm{~min}$, to $53 \%$ from 10 to $18 \mathrm{~min}$, to $57 \%$ from 18 to $23 \mathrm{~min}$, and to $85 \%$ from 23 to $28 \mathrm{~min}$. From 28 to $33 \mathrm{~min}$ the $\mathrm{ACN}$ was kept at $85 \%$. The solvent was delivered at a rate of $0.8 \mathrm{ml} / \mathrm{min}$. The UV spectrum was produced for each chromatographic peak generated. 12-Hydroxy-5,8,10-heptadecatrienoic acid (HHT) and 12-HETE were used as markers of the cyclooxygenase and the 12-lipoxygenase pathways, respectively.

Authentic standards of 12-HETE and HHT derived from platelets were provided by Dr. S. Hammarström (Department of Physiological Chemistry, Karolinska Institutet, Stockholm, Sweden). Identification of 12-HETE and HHT was based on cochromatography with authentic standards and on their UV spectrum.

\section{Platelet aggregation}

Aggregation was measured with a chronolog electronic aggregometer; $1 \mathrm{ml}$ of platelets $\left(2 \times 10^{8} / \mathrm{ml}\right)$ in $\mathrm{Ca}^{2+} / \mathrm{Mg}^{2+}$-free PBS was prewarmed to $37^{\circ} \mathrm{C}$ for $5 \mathrm{~min}$. Aggregation was initiated by adding thrombin $(1$ or $10 \mathrm{U} / \mathrm{ml}), \operatorname{ADP}(0.5 \mu \mathrm{g} / \mathrm{ml})$, or ristocetin $(1 \mathrm{mg} / \mathrm{ml})$ under continuous stirring. Two electrodes were placed in the platelet suspension, and aggregation was read as the increase in impedance as platelets accumulated on the electrodes. Results were expressed as the aggregation after $3 \mathrm{~min}$ divided by the maximal aggregation.

\section{$D N A$ synthesis of cultured human keratinocytes}

Primary cultures of adult human epidermal keratinocytes were established as previously described [13]. Ethanolic dilutions of 12-HETE (ethanol $<0.1 \%$ ) were added to confluent cultures for $72 \mathrm{~h}$. DNA synthesis was determined as $\left({ }^{3} \mathrm{H}\right)$ thymidine incorporation into DNA and as autoradiographic labeling indices, as previously described [16].

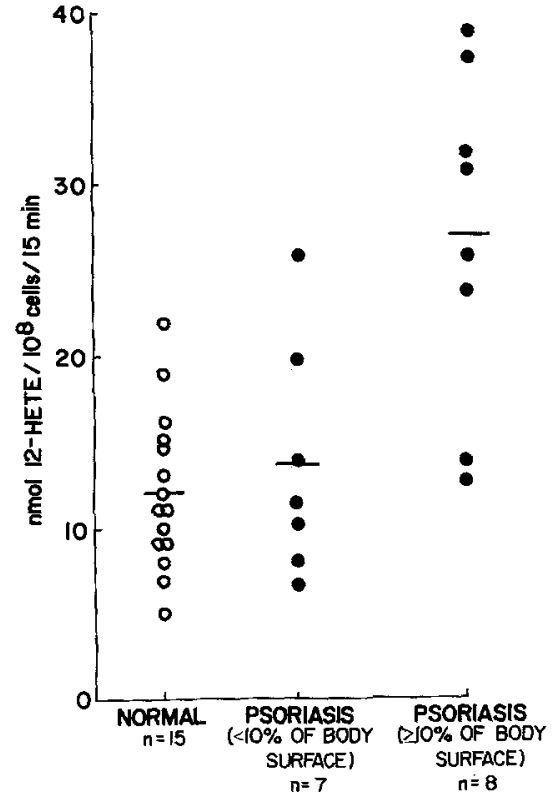

Fig. 1. Formation of 12-hydroxy-5,8,10,14-eicosatetraenoic acid (12-HETE) by platelets from normals and psoriatics. Two milliliters of platelets $\left(2 \times 10^{8} / \mathrm{ml}\right)$ was incubated with $2 \mu \mathrm{M}$ arachidonic acid and $1 \mu \mathrm{M}$ A23187 for $15 \mathrm{~min}$ at $37^{\circ} \mathrm{C}$. 12 HETE was isolated using reversed-phase high-performance liquid chromatography (RP-HPLC) and quantified by integrated optical density. The mean value is indicated by a horizontal bar. Normal vs. psoriasis ( $>10 \%$ of body surface), $P<0.01$

\section{Statistics}

Statistical significance was assessed using the Wilcoxon ranksum test for unpaired data. A $P$ value below 0.05 was considered significant.

\section{Results}

In Fig. 1 the formation of 12-HETE by normal and psoriatic platelets can be seen. Psoriatics with widespread diseases ( $\geqslant 10 \%$ of the body surface) formed on average $125 \%$ more $12-\mathrm{HETE}$ than normals, whereas patients with mild disease $(<10 \%$ of the body surface involved) had normal 12-lipoxygenase activity. The formation of HHT, a marker of cyclooxygenase activity, was also stimulated in widespread psoriasis (Fig. 2), although to a lesser degree (98\%) than $12-$ HETE.

Platelet aggregation was elicited by adding thrombin $(1 \mathrm{U} / \mathrm{ml})$. A typical response of normal and psoriatic platelets is shown in Fig. 3. Psoriatic platelets showed an increased rate of aggregation, but the maximal aggregation was normal. Expressing aggregation as the aggregation obtained after $3 \mathrm{~min}$, a shortened platelet aggregation time was present in both mild and widespread disease (Fig. 4). The aggregation of psoriatic platelets was also enhanced in response to a higher thrombin concentration $(10 \mathrm{U} / \mathrm{ml})$ 


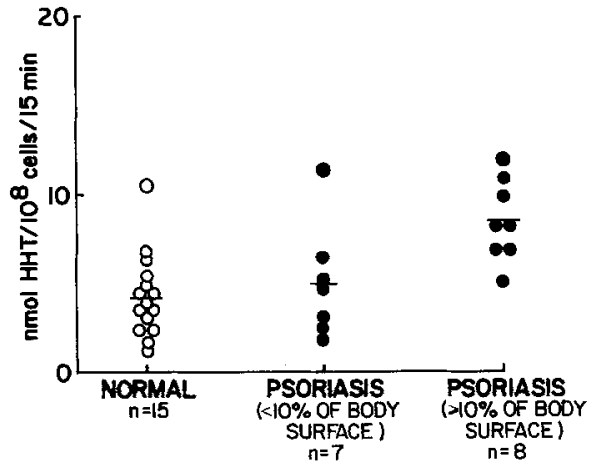

Fig. 2. Formation of 12-hydroxy-5,8,10-heptadecatrienoic acid (HHT) by platelets from normals and psoriatics. For explanation, see legend to Fig. 1. Normal vs. psoriasis $(>10 \%$ of body surface), $P<0.01$

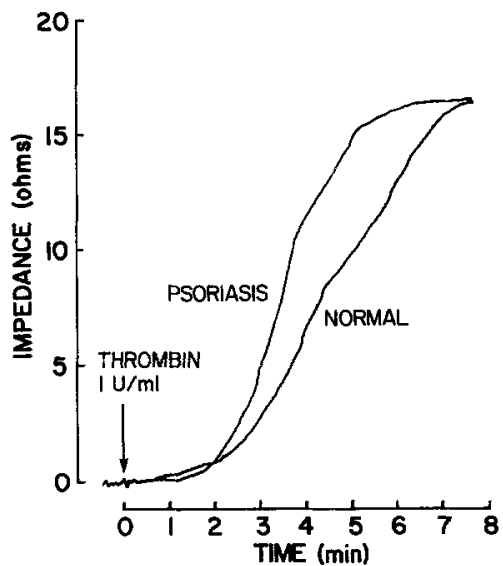

Fig. 3. Thrombin-induced $(1 \mathrm{U} / \mathrm{ml})$ aggregation of platelets from a normal volunteer and a patient with psoriasis. Aggregation was recorded as increase of impedance as a function of time

and to ADP $(0.5 \mu \mathrm{g} / \mathrm{ml})$ and ristocetin $(1 \mathrm{mg} / \mathrm{ml})$ (data not shown). Comparison of platelet cyclooxygenase activity (HHT) and platelet aggregation (i.e., aggregation after $3 \mathrm{~min} / \mathrm{maximal}$ aggregation) failed to show a significant correlation $(r=0.46, n=15, \mathrm{NS})$.

To assess whether platelet-derived 12-HETE modifies keratinocyte DNA synthesis, 12-HETE was added to cultured human keratinocytes (Fig. 5). Addition of 12-HETE resulted in a dose-dependent stimulation of $\left({ }^{3} \mathrm{H}\right)$ thymidine incorporation into DNA. At concentrations higher than $10^{-6} M$, no stimulation of the DNA synthesis was observed. The stimulation was maximal $(68 \%)$ at $10^{-7} \mathrm{M}$. At concentrations of $10^{-5} M$ or higher, the keratinocyte DNA was not affected by 12-HETE (Fig. 5).

The ability of 12-HETE to stimulate keratinocyte DNA synthesis was confirmed by determining autoradiographic labeling indices (data not shown).

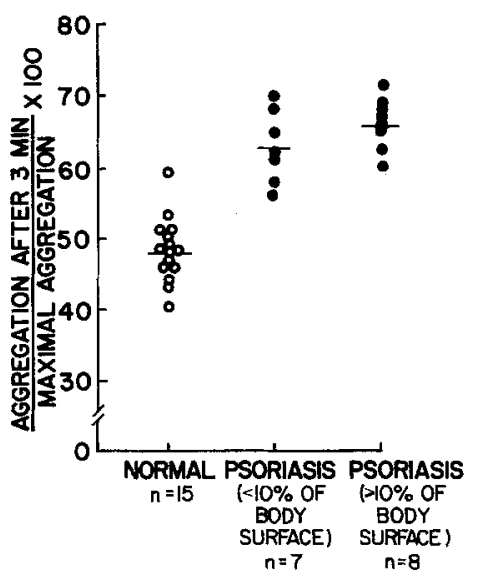

Fig. 4. Platelet aggregation induced by thrombin $(1 \mathrm{U} / \mathrm{ml})$ in normals and psoriatics. Aggregation was measured as the increase of impedance. The mean value is indicated by a horizontal bar. Normal vs. psoriasis ( $>10 \%$ of body surface), $P<0.01$

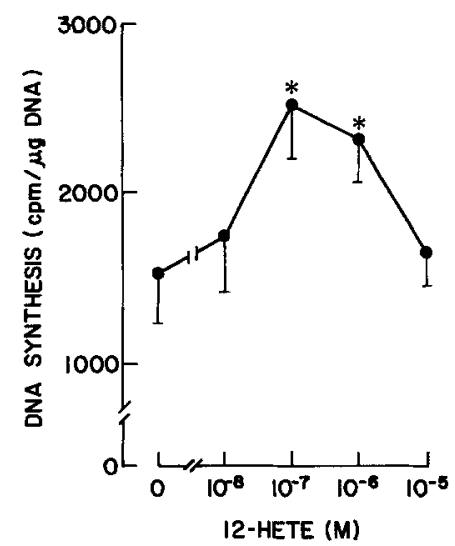

Fig. 5. Effect of platelet-derived 12-HETE on DNA synthesis of human epidermal keratinocytes in culture. Confluent cultures incubated with 12-HETE for $72 \mathrm{~h}$ were labeled with $1 \mu \mathrm{Ci} / \mathrm{ml}$ $\left({ }^{3} \mathrm{H}\right)$ thymidine $(50 \mathrm{Ci} / \mathrm{mmol})$ for the last $6 \mathrm{~h}$ of incubation. Results are expressed as the mean \pm SD of six experiments. 12HETE $\left(10^{-6} M\right.$ and $\left.10^{-7} M\right)$ vs. control, $P<0.01\left(^{*}\right)$

\section{Discussion}

From the results of this study, it appears that platelet aggregation and arachidonic acid metabolism are stimulated in psoriatics with active disease. Hyperaggregatory platelets have also been reported in patients with diabetes mellitus [5] and in cigarette smokers [16]. Furthermore, the 12-lipoxygenase activity of platelets can be stimulated by cigarette smoking [4]. Since both diabetics and smokers were excluded from our study, the increased platelet activities in the patients studied by us seem to be related to their psoriasis. However, other factors not controlled for in the study may also be involved.

The transformation of arachidonic acid via 12lipoxygenase was stimulated to a higher degree than 
via cyclooxygenase in psoriatic patients; 12lipoxygenase activity results in the formation of $12-$ HETE, which was shown to stimulate the DNA synthesis of cultured human keratinocytes. As observed with leukotrienes [14], the stimulatory effect of 12HETE on the DNA synthesis of keratinocyte cultures was only present at low concentrations. Combined with the ability of platelet-derived 12-HETE to induce an erythematous skin response characterized by neutrophil accumulation in humans [6], our findings are compatible with the idea that a 12-lipoxygenase product of platelets may participate in the pathophysiology of psoriasis. This increased 12-HETE formation by psoriatic platelets may explain why increased 12 HETE release has been reported in psoriatic neutrophil suspensions contaminated with platelets [22]. In addition, an increased platelet contamination may have contributed to these results.

Products of the 12-lipoxygenase pathway do not participate in the aggregation of platelets [21]. In contrast, certain cyclooxygenase products, particularly thromboxane $A_{2}$, are potent proaggregatory agents [8]. Release of thromboxane $A_{2}$ may be responsible for the enhanced platelet aggregation induced by thrombin in psoriasis. In addition, other metabolic pathways leading to platelet aggregation appear to be activated in psoriasis, because we found no significant correlation between cyclooxygenase activity and aggregation in psoriatic platelets. Recently, psoriatic patients have been found to have increased whole blood viscosity [23], enhanced spontaneous platelet aggregation [1], and enhanced platelet regeneration time [1]. Combined with the stimulated platelet activities that we found, these results indicate that psoriatics may be prone to develop occlusive vascular disease. These experimental data are in accordance with a retrospective study in which the incidence of occlusive vascular disease was higher in psoriatic patients than in nonpsoriatic dermatological patients [19]. It should, however, be borne in mind that carefully designed prospective studies are required to decide not only whether occlusive vascular disease is in fact more common in psoriatics, but also to control for other conditions that may be associated with an increased risk of thrombosis.

On a molar basis 12-HETE was 100-1000 times less potent than leukotriene $B_{4}[14]$ in stimulating DNA synthesis of cultured human keratinocytes. The ratio of 12-HETE to leukotriene $B_{4}$ in psoriatic lesions is, however, of the same order of magnitude. As a consequence, the biological effects of 12-HETE in psoriatic lesions may be as pronounced as those of leukotriene $\mathrm{B}_{4}$. Epidermis itself is also capable of synthesizing 12-HETE $[11,15]$, although there is recent evidence that 12 -HETE present in psoriatic epidermis may be stereochemically different from platelet-derived 12-HETE [24]. In the future, therefore, it would be of interest to determine whether epidermis-derived 12-HETE also possesses mitogenic activity. Platelets contain other mitogenic factors than 12-HETE [20]. Whether these mitogens also affect epidermal keratinocyte proliferation is not known.

Although this study has demonstrated abnormalities of peripheral platelets from patients with psoriasis, a key question is to what extent platelets or their products are present in lesional psoriatic skin. In electron microscopic studies, the microvascular system of psoriatic skin displays various abnormalities, consisting of dilatation and convolution of the capillary loops with endothelial gaps [3]. Thrombosis is, however, not part of the histopathological picture of psoriasis, and platelets are apparently not plentiful in psoriatic lesions [3]. As mentioned above, it may now be possible to make a distinction between epidermal and platelet-derived 12-HETE. Using platelet-specific 12-HETE as a marker of platelets, it may, therefore, be possible to decide whether platelets are involved in the psoriatic skin lesion. Also, it remains to be determined whether the platelet abnormalities are specific for psoriasis or shared by other inflammatory dermatoses.

\section{References}

1. Berrettini M, Parise P, Costantini V, Grasselli S, Nenci GG (1985) Platelet activation in psoriasis. Thromb Haemost 53:195-197

2. Borgeat P, Samuelsson B (1979) Arachidonic acid metabolism in polymorphonuclear leukocytes: effects of ionophore A23187. Proc Natl Acad Sci USA 76:21482151

3. Braverman IM (1972) Electron microscopic studies of the microcirculation in psoriasis. J Invest Dermatol 59:91-98

4. Chang W-C, Fukuda S, Tai H-H (1983) Cigarette smoking stimulates lipoxygenase but not cyclooxygenase pathway in platelets. Biochim Biophys Acta 115:499-505

5. Davi G, Reni GB, Averna $M$, Novo S, DiFede, Mattina A, Notarbartolo A, Strano A (1982) Enhanced platelet release reaction in insulin-dependent and insulin-independent diabetic patients. Haemostasis 12:275-281

6. Dowd PM, Kobza Black A, Woollard PM, Camp RDR, Greaves MW (1985) Cutaneous responses to 12-hydroxy5,8,10,14-eicosatetraenoic acid. J Invest Dermatol 84:537541

7. Goetzl EJ, Woods JM, Gorman RR (1977) Stimulation of human eosinophil and neutrophil polymorphonuclear leukocyte chemotaxis and random migration by $12-\mathrm{I}$ hydroxy-5,8,10,14-eicosatetraenoic acid. J Clin Invest 59: $179-183$

8. Hamberg M, Svensson J, Samuelsson B (1975) A new group of biologically active compounds derived from prostaglandin endoperoxides. Proc Natl Acad Sci USA 72:2994-2998

9. Hamberg M, Svensson J, Samuelsson B (1976) Prostaglandin endoperoxides. A new concept concerning the mode of action and release of prostaglandins. Proc Natl Acad Sci USA $71: 3824-3828$ 
10. Hammarström S, Hamberg M, Samuelsson B, Duell EA, Stawiski M, Voorhees JJ (1975) Increased concentrations of free arachidonic acid, prostaglandin $\mathrm{E}_{2}$ and $\mathrm{F}_{2}$ and of $12-\mathrm{L}-$ hydroxy-5,8,10,14-eicosatetraenoic acid (HETE) in epidermis of psoriasis: evidence of perturbed regulation of arachidonic acid levels in psoriasis. Proc Nati Acad Sci USA $72: 5130-5134$

11. Hammarström S, Lindgren JA, Marcelo CL, Duell EA, Anderson TF, Voorhees J (1979) Arachidonic acid transformations in normal and psoriatic skin. J Invest Dermatol $73: 180-183$

12. Kragballe K, Voorhees JJ (1983) Arachidonic acid and leukotrienes in dermatology. J Invest Dermatol 81:293296

13. Kragballe K, Desjarlais L, Marcelo CL (1985) Increased DNA synthesis of uninvolved psoriatic epidermis is maintained in vitro. Br J Dermatol 112:263-270

14. Kragballe K, Desjarlais L, Voorhees JJ (1985) Leukotrienes $B_{4}, C_{4}$, and $D_{4}$ stimulate DNA synthesis of cultured human epidermal keratinocytes. Br I Dermatol 113:43-52

15. Kragballe K, Desjarlais L, Altman DA, Voorhees JJ (1986) Uninvolved psoriatic epidermis has increased capacity to synthesize 12-hydroxy-eicosatetraenoic acid. J Invest Dermatol 87:47-52

16. Levine PH (1973) An acute effect of cigarette smoking on platelet function. A possible link between smoking and arterial thrombosis. Circulation $48: 619-623$
17. Luderer JR, Riley DL, Demers LM (1983) Rapid extraction of arachidonic acid metabolites utilizing octadecyl reversephase columns. J Chromatogr 273:402-409

18. Marcelo CL, Kim YG, Kaine JL, Voorhees JJ (1978) Stratification, specialization, and proliferation of primary keratinocyte cultures. J Cell Biol 79:356-370

19. McDonald CJ, Calabresi P (1978) Psoriasis and occlusive vascular disease. Br J Dermatol 99:469-475

20. Ross R (1981) Platelet-derived growth factor. In: Baserga $R$ (ed) Handbook of experimental pharmacology, vol 57. Springer, New York Berlin Heidelberg, pp 133-159

21. Saurs AR, Sprecher H, Saukarappa SK, Needleman P (1982) Selective inhibitors of platelet arachidonic acid metabolism: independent of lipoxygenase. In: Samuelsson B, Paoletti R (eds) Leukotrienes and other lipoxygenase products, vol 9. Raven, New York, pp 19-28

22. Walker JR, Littlewood SM, Dawson W, Allen BR (1983) Metabolism of $\left(1-{ }^{14} \mathrm{C}\right)$ arachidonic acid by peripheral blood cells from psoriatic patients. J Invest Dermatol 80:359

23. Wolf R, Machtey I, Feuerman EJ, Creter D (1981) Blood hyperviscosity in psoriasis. Acta Derm Venereol (Stockh) $61: 153-154$

24. Woollard PM (1985) Stereochemical difference between 12hydroxy-5,8,10,14-eicosatetraenoic acid (12-HETE) in platelets and psoriatic lesions. J Invest Dermatol 84:455

Received December 6, 1985 